\title{
Erratum to: Injurious mechanical ventilation affects neuronal activation in ventilated rats
}

María Elisa Quilez ${ }^{1,2,5}$, Gemma Fuster ${ }^{1,2}$, Jesús Villar ${ }^{1,3}$, Carlos Flores ${ }^{1,4}$, Octavi Martí-Sistac ${ }^{1,2,5}$, Lluís Blanch ${ }^{1,2}$ and Josefina López-Aguilar ${ }^{1,2^{*}}$

Unfortunately, the original version of this article [1] contained an error. Affiliation no. 5 had been omitted to be included for the first author, María Elisa Quilez. This has now been corrected. The full list of affiliations for the first author are:

María Elisa Quilez ${ }^{1,2,5}$

1 CIBER de Enfermedades Respiratorias, Instituto de Salud Carlos III. C/ Sinesio Delgado 6, Madrid, 28029, Spain

2 Critical Care Center, Corporació Sanitaria Parc Taulí, Institut Universitari, Esfera UAB. Parc Taulí sn. Sabadell, 08208, Spain

5 Universitat Autònoma de Barcelona. Campus de la UAB, Bellaterra, 08193, Spain

\section{Author details}

${ }^{1}$ CIBER de Enfermedades Respiratorias, Instituto de Salud Carlos III, C/ Sinesio Delgado 6, Madrid 28029, Spain. ${ }^{2}$ Critical Care Center, Corporació Sanitaria Parc Taulí, Institut Universitari, Esfera UAB. Parc Taulí sn, Sabadell 08208, Spain. ${ }^{3}$ Research Unit, Hospital Universitario Dr.Negrín, Barranco de la Ballena s/n, Las Palmas de Gran Canaria 35010, Spain. ${ }^{4}$ Research Unit, Hospital Universitario N.S. de Candelaria, Carretera del Rosario 145, Santa Cruz de Tenerife 38010, Spain. ${ }^{5}$ Universitat Autònoma de Barcelona, Campus de la UAB, Bellaterra 08193, Spain.

Received: 17 September 2015 Accepted: 23 September 2015 Published online: 27 October 2015

\section{Reference}

1. Quilez ME, Fuster G, Villar J, Flores C, Martí-Sistac O, Blanch L, et al.

Injurious mechanical ventilation affects neuronal activation in ventilated rats. Crit Care. 2011;15:R124.

\footnotetext{
* Correspondence: jlopeza@tauli.cat

${ }^{1}$ CIBER de Enfermedades Respiratorias, Instituto de Salud Carlos III, C/ Sinesio

Delgado 6, Madrid 28029, Spain

${ }^{2}$ Critical Care Center, Corporació Sanitaria Parc Taulí, Institut Universitari,

Esfera UAB. Parc Taulí sn, Sabadell 08208, Spain

Full list of author information is available at the end of the article
} 